\title{
Use of azithromycin as treatment against Chlamydia trachomatis?
}

\author{
José Luis Maldonado-Calderón, Francisco Carlos López-Márquez and Pablo Ruiz-Flores
}

Universidad Autónoma de Coahuila, Center of Biomedical Research, Coahuila, Mexico

\begin{abstract}
Azithromycin and doxycycline effectiveness has been demonstrated in the treatment of urogenital chlamydiasis, which has remained unchanged for a long time. Autoinoculation has been proposed as a method of reinfection and persistence of the disease in women and probably also owing to azithromycin pharmacokinetics in this tissue. With the new diagnostic methods and tests of cure, a difference has been demonstrated in favor of doxycycline in the treatment of rectal chlamydiasis Antimicrobial resistance has not played a relevant role since no treatment-resistant strains have been found in vivo. Nevertheless, azithromycin remains a first-choice drug, since it can be administered as a single dose, which favors therapeutic adherence.
\end{abstract}

KEY WORDS: Chlamydia trachomatis. Azithromycin. Test of cure.

\section{Introduction}

Chlamydia infection caused by Chlamydia trachomatis (genotypes A-K) constitutes the most reported disease and is among the most prevalent sexually transmitted infections in the United States; the age group of 15 to 24 years is the most affected.1., According to the World Health Organization, there is an annual estimate of 131 million new infections caused by Chlamydia trachomatis. ${ }^{3}$

The disease is asymptomatic in $70 \%$ of women and $50 \%$ of men. ${ }^{4}$ When it produces symptoms, women often show changes in vaginal discharge, intermenstrual bleeding or dyspareunia (data suggestive of cervicitis); on physical examination, cervical erythema, friable cervix and endocervical discharge are identified. In addition, symptoms can mimic urinary tract infection. In men, it occurs more commonly as urethritis (dysuria, discomfort and urethral discharge) and on physical examination, irritation or erythema of the urinary meatus and sometimes urethral discharge (which tends to be mucoid and not purulent) are observed; it can also occur in the form of orchitis or epididymitis. In women, it can cause serious sequelae such as pelvic inflammatory disease, ectopic pregnancy, miscarriage and infertility. ${ }^{5,6}$

To establish the diagnosis of urogenital chlamydia, there are different methods such as cell culture, rapid diagnostic test, serum antibodies against chlamydia and nucleic acid amplification test (NAAT), which is the method of choice, since it reaches the highest sensitivity and a specificity that is comparable to that of cell culture and, in addition, the World Health Organization recommends it. The ideal sample to perform the NAAT in men is the first jet of the first urine of the day, while in women it is that obtained by vaginal smear. ${ }^{4,6}$

The World Health Organization and the Centers for Disease Control and Prevention (CDC) suggest $1 \mathrm{~g}$ of oral azithromycin in a single dose or $100 \mathrm{mg}$ of oral doxycycline twice daily for 7 days for the treatment of chlamydia. ${ }^{4,7}$

\section{Azithromycin}

Azithromycin is an antibiotic from the family of macrolides, a semisynthetic derivative of erythromycin. ${ }^{8}$ It expresses its antibiotic activity by binding to the $50 \mathrm{~S}$ ribosomal subunit (rRNA 23S) and inhibits protein
Correspondence:

Francisco Carlos López-Márquez

E-mail: francisco.c.lopezmarquez@gmail.com
Date of reception: 09-05-2017

Date of acceptance: 30-08-2017

DOI: 10.24875/GMM.M19000211
Gac Med Mex. 2018;154:589-592

Contents available at PubMed www.gacetamedicademexico.com 
synthesis by preventing the formation of the peptide bond between the amino acids of the nascent protein. ${ }^{9}$ Its pharmacokinetic profile is characterized by a rapid and extensive uptake from circulation to intracellular compartments (including the so-called inflammatory cells); subsequently, it is released little by little where it is needed, which allows a single-dose regimen or one dose for a single day. ${ }^{9-11}$

\section{Azithromycin or doxycycline?}

As previously mentioned, for uncomplicated urogenital chlamydia, the World Health Organization and the CDCs recommend $1 \mathrm{~g}$ of oral azithromycin as a single dose or $100 \mathrm{mg}$ oral doxycycline twice daily for seven days. This recommendation is based on a meta-analysis of 12 clinical trials on azithromycin versus doxycycline for the treatment of urogenital chlamydia carried out in 2002," which demonstrated that both are equally efficacious, with rates of microbial cure of 97 and $98 \%$ for azithromycin and doxycycline, respectively. However, in 11 of those clinical trials, less sensitive diagnostic and follow-up methods were used to monitor cure (culture and immunoassays) than those that are currently recommended (NAAT), which may have caused underestimation of the ranges of treatment failure. ${ }^{7,12,13}$ In a new meta-analysis carried out in 2014, it was concluded that there is an efficacy greater than $3 \%$ in favor of doxycycline for the treatment of urogenital chlamydia and of approximately $7 \%$ for the treatment of symptomatic urethral infection in men. ${ }^{13}$

\section{Rectal chlamydia}

The difference in efficacy between azithromycin in comparison with doxycycline for the treatment of rectal chlamydia may be greater than that reported in urogenital chlamydia. . $^{14,15}$ In 2015 an efficacy of $83 \%$ was recorded for azithromycin and higher than $99 \%$ for doxycycline in the treatment of rectal infection by chlamydia, although the quality of evidence was acknowledged to be low. ${ }^{16}$ Nevertheless, an approximate effectiveness of $83 \%$ for azithromycin is much lower than $97.4 \%$ indicated in the treatment of urogenital chlamydia infection. ${ }^{14}$

This might be due to the bioavailability of azithromycin in rectal tissue, and it is unknown if it is similar to that of urethral and cervical mucosa. Some experimental studies in animals, which investigate chlamydia in the large intestine, show a lack of local immune response, as well as an absence of polymorphonuclear cells, ${ }^{17}$ and given that azithromycin uses these cells to reach the site of infection, the lack of these and a decreased immune reaction in the rectum might decrease azithromycin effectiveness. In 2013, the inflammatory response to chlamydia rectal infection was examined, with suppressed inflammatory cytokines being reported in HIV-negative subjects infected with chlamydia, which provides further data and supports the aforementioned. ${ }^{18}$

In addition to all this, chlamydia autoinoculation from the gastrointestinal to the urogenital tract in women has been proposed, and it has acquired relevance recently. Thus, the gastrointestinal tract has been mentioned as a persistent infection niche..$^{17}$ In 2015, using a mathematical model, the probability was estimated for a woman with chlamydia genital infection treated either with azithromycin or doxycycline to remain chlamydia-free when the possibility of autoinoculation was taken into account; it was demonstrated that when the probability of autoinoculation was assumed to be $100 \%$, the likelihood for the patient to remain free of genital infection after treatment with doxycycline was 96.8 and $81.9 \%$ with azithromycin; that is, 3.2 and $18.1 \%$ of probability, respectively, not to eliminate the infection, 5.7 times more chance for not eliminating the infection with azithromycin ion comparison with doxycycline. Furthermore, if the patient had rectal infection, the probability of remaining free of genital infection after the treatment with azithromycin was 78.2 to $94.3 \%$, and after treatment with doxycycline from 96.7 to $97.1 \%$, which corresponds to 2 to 6.6 times higher probability not to eliminate the chlamydia infection with azithromycin in comparison with doxycycline. ${ }^{15}$

Currently, the CDCs recommend as treatment for rectal chlamydia the same than that for urogenital chlamydia; however, this is nothing but an extrapolation of the findings on similar efficacy (which is questioned) of both drugs in the treatment of urogenital chlamydia. In an analysis performed in 2015 at a clinic for sexually transmitted diseases in male patients diagnosed with rectal chlamydia between 1993 and 2012, men treated with azithromycin were found to have a significantly higher risk of persistence/recurrence of rectal chlamydia in comparison with subjects treated with doxycycline (similar results were obtained in a retrospective review carried out in 2016 19 ); in addition, the authors suggest that a 7-day doxycycline regimen may be superior to an azithromycin $1 \mathrm{~g}$ single dose for the treatment of rectal chlamydia infection. ${ }^{20}$ Another study suggests that $100 \mathrm{mg}$ of doxycycline twice daily are highly effective for asymptomatic rectal chlamydia, with bacterial cure (as assessed by NAAT) being achieved in $98.8 \%$ of cases. ${ }^{21}$ 


\section{Antimicrobial resistance}

Bacterial resistance to antibiotics has been proposed as a cause of treatment failure. Given the complexities of Chlamydia trachomatis life cycle, ${ }^{22,23}$ it is possible that persistent forms, which are involved in heterotypic resistance, may require several weeks to restore detectable replicative infection after treatment with a single dose of azithromycin. ${ }^{24}$ However, only heterotypic resistance (which occurs when an infection has a small proportion of resistant organisms among a susceptible population) has been possible to be documented, with mutations in 23S rRNA being associated with in vitro resistance to macrolides. So far, no strains with homotypic resistance (where the entire population of organisms survives after treatment) have been identified. ${ }^{25,26}$

\section{False-positive test-of-cure}

NAAT is the method of choice to perform a "test-ofcure". Unfortunately this method only provides information about the presence of genetic material, but not on viability of the pathogen or if it's still infectious; therefore, intermittent positive results could be the result of viable or non-viable genetic material deposition from a sexual partner, release of degraded cells genetic material or elementary bodies containing Chlamydia trachomatis genetic material. ${ }^{27} \mathrm{~A}$ negative NAAT may indicate an eliminated infection; however, it also can reflect persistent infection. ${ }^{28}$

Currently, the guidelines recommend a test of cure 3 months after treatment conclusion, instead of performing it 4 weeks after having received it, in order to minimize the risk of false-positive diagnoses. ${ }^{25}$ False-positive results can occur three weeks after treatment due to DNA persistence, and because persistent disease takes a few weeks to emerge. Tests of cure should be deferred for at least 5 weeks post-treatment. ${ }^{22,27}$ In addition, it has been shown that, in order to prevent reinfection, women should refrain from having intercourse without a condom or, in any case, use a condom in every sexual intercourse for at least one month after having received the treatment. ${ }^{29}$

\section{Conclusion}

Even when efficacy in the treatment of urogenital chlamydia has been shown to be slightly higher favor of doxycycline, azithromycin remains a medication of choice, since it can be administered as a single dose, with higher therapeutic adherence being reached.
However, if gastrointestinal tract autoinoculation is taken into account as a cause of treatment failure, doxycycline should be preferred, since azithromycin pharmacokinetics in rectal tissue is unknown.

In addition, the CDCs recommends the test of cure only if treatment adherence is questionable, if symptoms persist or if reinfection is suspected. Its performance is not recommended if the right treatment was given in the right way; however, given that most urogenital or rectal chlamydia infections are asymptomatic, higher therapeutic efficacy against rectal chlamydia infection has been shown when using doxycycline in comparison with azithromycin, if we take the possibility of autoinoculation into account, we should always suspect reinfection, and every patient should therefore have a test of cure practiced.

This strengthens the reconsideration of urogenital chlamydia treatment and taking rectal infection with chlamydia into account as a source of autoinoculation. In addition, it is advisable to stratify the populations at risk of suffering rectal chlamydia, as well as obtaining genital and rectal samples from patients in whom urogenital chlamydia is suspected.

It is desirable for randomized controlled trials comparing azithromycin efficacy against doxycycline for the treatment of urogenital and rectal chlamydia infection to be conducted.

\section{References}

1. Centers for Disease Control and Prevention. Sexually transmitted disease surveillance 2015. EE. UU.: Department of Health and Human Services; 2016.

2. Centers for Disease Control and Prevention. CDC Fact Sheet. Reported STDs in the United States 2015 National Data for Chlamydia, Gonorrhea, and Syphilis. EE. UU.: Department of Health and Human Services; 2016.

3. World Health Organization. Report on global sexually transmitted infection surveillance 2015. Ginebra, Suiza: World Health Organization; 2016.

4. World Health Organization. WHO Guidelines for the Treatment of Chlamydia trachomatis. Ginebra, Suiza: World Health Organization; 2016.

5. Lane AB Decker CF. Chlamydia trachomatis infections. Dis Mon. 2016;62:269-273

6. Ahmadi A, Khodabandehloo M, Ramazanzadeh R, Farhadifar F, Roshani D, Ghaderi E, et al. The relationship between Chlamydia trachomatis genital infection and spontaneous abortion. J Reprod Infertil. 2016;17:110-116.

7. Meyer T. Diagnostic procedures to detect chlamydia trachomatis infections. Microorganisms. 2016;4:25.

8. Workowski KA, Bolan GA, Centers for Disease Control and Prevention. Sexually transmitted diseases treatment guidelines, 2015. MMWR Recomm Rep. 2015;64:1-137.

9. Bakheit A, Al-Hadiya B, Abd-Elgalil A. Azithromycin. En: Brittain H. Profiles of drug substances, excipients and related methodology. EE. UU.: Academic Press; 2014.

10. Cobos-Trigueros N, Ateka O, Pitart C, Vila J. Macrólidos y cetólidos. Enf Infec Microb Clin. 2009;27:412-418.

11. Jelić $D$, Antolović R. From erythromycin to azithromycin and new potential ribosome-binding antimicrobials. Antibiotics (Basel). 2016;5:29.

12. Lau CY, Qureshi AK. Azithromycin versus doxycycline for genital chlamydial infections: a meta-analysis of randomized clinical trials. Sex Transm Dis. 2002;29:497-502.

13. Geisler W, Uniyal A, Lee J, Lensing S, Johnson S, Perry R et al. Azithromycin versus doxycycline for urogenital chlamydia trachomatis infection. New Engl J Med. 2015;373:2512-2521. 
14. Kong FY, Tabrizi SN, Law M, Vodstrcil LA, Chen M, Fairley C, et al. azithromycin versus doxycycline for the treatment of genital chlamydia infection: a meta-analysis of randomized controlled trials. Clin Infect Dis. 2014;59:193-205.

15. Craig A, Kong FY, Yeruva L, Hocking JS, Rank RG, Wilson DP, et al. Is it time to switch to doxycycline from azithromycin for treating genita chlamydial infections in women? Modelling the impact of autoinoculation from the gastrointestinal tract to the genital tract. BMC Infect Dis. 2015;15:200.

16. Kong FY, Tabrizi SN, Fairley CK, Vodstrcil LA, Huston WM, Chen M, et al. The efficacy of azithromycin and doxycycline for the treatment of rectal chlamydia infection: a systematic review and meta-analysis. J Antimicrob Chemother. 2015;70:1290-1297.

17. Rank RG, Yeruva L. Hidden in plain sight: chlamydial gastrointestinal infection and its relevance to persistence in human genital infection. Infect Immun. 2014;82:1362-1371.

18. Heiligenberg M, Lutter R, Pajkrt D, Adams K, De-Vries H, Heijman T, et al. Effect of HIV and chlamydia infection on rectal inflammation and cytokine concentrations in men who have sex with men. Clin Vaccine Immunol. 2013;20:1517-1523.

19. Gratrix J, Brandley J, Dane M, Plitt S, Smyczek P, Read R, et al. A retrospective review of treatment failures using azithromycin and doxycycline in the treatment of rectal chlamydia infections in women and men who have sex with Men. Sex Transm Dis. 2016;43:110-112.

20. Khosropour CM, Dombrowski JC, Barbee LA, Manhart L, Golden MR Comparing azithromycin and doxycycline for the treatment of rectal chlamydial infection: a retrospective cohort study. Sex Transm Dis. 2014;41:79-85
21. Horner PJ. Azithromycin antimicrobial resistance and genital chlamydia trachomatis infection: duration of therapy may be the key to improving efficacy. Sex Transm Infect. 2012;88(3):154-156

22. Ziklo N, Huston W, Hocking J, Timms P. Chlamydia trachomatis genita tract infections: when host immune response and the microbiome collide. Trends Microbiol. 2016;24:750-765.

23. Cossé MM, Hayward RD, Subtil A. One face of chlamydia trachomatis: the infectious elementary body. Curr Top Microbiol Immunol. 2018:412:35-58.

24. Elgalib A, Alexander S, Tong C, White JA. Seven days of doxycycline is an effective treatment for asymptomatic rectal chlamydia trachomatis infection. Int J STD AIDS. 2011;22:474-477.

25. Kong FY, Hocking JS. Treatment challenges for urogenital and anorectal chlamydia trachomatis. BMC Infect Dis. 2015;15:293.

26. Madhivanan P, Krupp K. Antibiotic resistance in prevalent bacterial and protozoan sexually transmitted infections. Indian J Sex Transm Dis AIDS. 2015;36:3.

27. Wind C, Schim Van Der Loeff MF, Unemo M, Schuurman R, Van-Dam AP, De-Vries $\mathrm{H}$. Time to clearance of chlamydia trachomatis RNA and DNA after treatment in patients coinfected with Neisseria gonorrhoeae: a prospective cohort study. BMC Infect Dis. 2016;16:554.

28. Dukers-Muijrers N, Morré SA, Speksnijder A, Van Der Sande M, Hoebe C. Chlamydia trachomatis test-of-cure cannot be based on a single highly sensitive laboratory test taken at least 3 weeks after treatment. PLoS One. 2012;7(3):e34108.

29. Lazenby GB, Korte JE, Tillman S, Brown FK, Soper DE. A recommendation for timing of repeat chlamydia trachomatis test following infection and treatment in pregnant and nonpregnant women. Int J STD AIDS. 2016;28:902-909. 